\title{
Le nain, le médecin et le divin marquis
}

Folie et Politique à Charenton entre le Directoire et l'Empire

The Dwarf, the Doctor, and the Divine Marquis Madness and Politics in

Charenton between the Directory and the Empire

Jean-Luc Chappey

\section{(2) OpenEdition \\ Journals}

\section{Édition électronique}

URL : https://journals.openedition.org/ahrf/12961

DOI : 10.4000/ahrf.12961

ISSN : 1952-403X

Éditeur :

Armand Colin, Société des études robespierristes

Édition imprimée

Date de publication : 1 décembre 2013

Pagination : 53-83

ISBN : 978290832789

ISSN : 0003-4436

\section{Référence électronique}

Jean-Luc Chappey, "Le nain, le médecin et le divin marquis », Annales historiques de la Révolution française [En ligne], 374 | octobre-décembre 2013, mis en ligne le 01 décembre 2016, consulté le 01 juillet 2021. URL : http://journals.openedition.org/ahrf/12961; DOI : https://doi.org/10.4000/ahrf. 12961 


\title{
LE NAIN, LE MÉDECIN ET LE DIVIN MARQUIS FOLIE ET POLITIQUE À CHARENTON ENTRE LE DIRECTOIRE ET L'EMPIRE
}

Jean-Luc CHAPPEY

\begin{abstract}
L'histoire de la folie sous la Révolution française a suscité l'intérêt sans forcément trouver sa place dans les débats historiographiques. Trop souvent considéré comme le produit de travaux menés par les historiens des sciences ou de la médecine, ce terrain reste encore ignoré par les spécialistes de l'histoire politique et sociale de la période, en dépit du succès de quelques productions récentes. Les évolutions des théories et des pratiques thérapeutiques, les transformations sociales et institutionnelles qui caractérisent le milieu des aliénistes ne relèvent pas seulement d'une histoire de la médecine mais touchent ainsi de près aux respirations politiques. Si une histoire politique de la folie n'est possible qu'en prenant précisément en compte les changements et les ruptures politiques, elle doit, en retour, permettre d'en préciser la nature et la portée. C'est dans cette perspective que notre attention a été captée par la Maison de Charenton et son directeur entre 1797 et 1814, FrançoisSimonet Coulmier (1741-1818). En tentant de questionner à nouveaux frais l'histoire mouvementée de cette institution où séjourneront un temps le marquis de Sade et le chansonnier Théodore Desorgue, il convient de mettre au jour les batailles politiques qui se jouent autour de la folie et d'en mesurer les enjeux sur l'héritage politique de l'idéal révolutionnaire dans les premières décennies du $\mathrm{XIX}^{\mathrm{e}}$ siècle $^{1}$.
\end{abstract}

Mots-clés : Folie, Charenton, Directoire, Coulmier, Royer-Collard, Sade

(1) Cet article est largement issu d'un travail commun mené en complicité avec Judith Lyon-Caen (CRH-Ehess). Qu'elle soit remerciée pour tous ses conseils. 
La réédition en 2007 de l'ouvrage de Marcel Gauchet et Gladys Swain, La pratique de l'esprit humain ${ }^{2}$, puis, plus récemment, l'écho favorable obtenu par le livre de Laure Murat, L'homme qui se prenait pour Napoléon ${ }^{3}$, invitent à replacer l'histoire de la folie au cœur des enjeux politiques de la Révolution française. Les travaux désormais classiques de Michel Foucault avaient clairement montré comment, depuis le XVII siècle, la folie s'était imposée comme une question relevant de logiques politiques et administratives, participant plus précisément au renforcement de l'encadrement de l'État monarchique sur les populations. Dans cette perspective, la période révolutionnaire et le geste mythique de la libération des fous accompli par Philippe Pinel (1745-1826) constituaient une rupture majeure dans l'Histoire de la folie ${ }^{4}$. La thèse du «Grand Renfermement » a pu être discutée, voire critiquée : Gauchet et Swain ont ainsi tenté de renverser ce mythe en montrant, au contraire, comment la Révolution française a pu être à l'origine d'une nouvelle conception de l'asile. Il n'en reste pas moins que la folie constitue, aux côtés du suicide $^{5}$, une des questions majeures qui s'impose, à la croisée des débats scientifiques, juridiques ou moraux, au cœur des dynamiques politiques de la période ${ }^{6}$.

La folie et les débats qu'elle suscite constituent en effet un point d'achoppement qui révèle, aux yeux de l'historien, des mutations essentielles non seulement concernant la position des médecins dans l'espace politique, mais surtout dans l'ordre des principes dont se revendique le régime politique. Dans cette perspective, François-Simonet Coulmier (1741-1818) et la Maison de Charenton qu'il dirige entre 1794 et 1817, ne sont pas méconnus, loin s'en faut. Ils bénéficient même d'un intérêt privilégié, soit, comme chez Gauchet et Swain, parce qu'ils apparaissent

(2) Marcel GaucheT et Gladys SwaIn, La pratique de l'esprit humain [1980], Paris, Gallimard, TEL, 2007.

(3) Laure MURAT, L'Homme qui se prenait pour Napoléon. Pour une histoire de la folie, Paris, Gallimard, 2011.

(4) Michel FouCAult Histoire de la folie à l'âge classique [1961 puis 1972], Paris, Gallimard, TEL, 1984 ; Robert CASTEL, L'Ordre psychiatrique. L'âge d'or de l'aliénisme, Paris, Éditions de Minuit, 1976 ; Jacques PosTEL et Claude QUÉTEL (dir.), Nouvelle histoire de la psychiatrie, Toulouse, Privat, 1983

(5) Voir le travail récent de Dominique GodINEAU, S'abréger les jours. Le suicide au XVIII siècle, Paris, Armand Colin, 2012.

(6) Sur les rapports entre folie et justice, Nathalie RoBATEL (dir), Le citoyen fou, Paris, PUF, 1991 ; Marc RÉNEVILLE, Crime et folie. Deux siècles d'enquêtes médicales et judiciaires, Paris, Fayard, 2003 ; Laurence GUIGNARD, Juger la folie. La folie criminelle devant les Assises au XIX ${ }^{e}$ siècle, Paris PUF, coll. « Droit et Justice », 2010. 
comme des formes repoussoirs et archaïques face au nouveau de la médecine "moderne", soit, à l'inverse, chez Laure Murat, parce qu'ils sont les symboles d'une forme de subversion salvatrice face à la mise en ordre opérée par le «pouvoir » médical. De part et d'autre, Coulmier est institué en représentant d'un monde révolu. La réinscription dans les logiques politiques de la trajectoire de ce dernier et de son échec dans la lutte qu'il mène contre Antoine-Athanase Royer-Collard (1768-1825), le médecin-chef de Charenton à partir de 1806 (et frère du député Pierre-Paul), doit permettre, non de réhabiliter une figure qui souffre d'une indéniable mauvaise réputation, mais de mieux comprendre les enjeux d'une lutte dont les mécanismes sont trop souvent appréhendés de manière réductrice, soit parce qu'on se contente de reprendre les jugements négatifs construits à l'époque, soit parce qu'on cherche à plaquer sur Coulmier des questions trop actuelles. Dans l'un et l'autre cas, on manque assurément ce qui fait son originalité. Pourquoi Coulmier a-t-il donc aussi mauvaise réputation ? S'il est indéniable qu'il souffre de la concurrence de la personnalité emblématique de Philippe Pinel, puis d'Étienne Esquirol qui lui succède... cela ne peut fournir une réponse satisfaisante.

\section{Soigner la folie à Paris sous le Directoire}

Membre de la Société royale de médecine, partisan de Mesmer dans les années 1780, nommé en 1793 médecin chef de l'hôpital de Bicêtre, Philippe Pinel constitue une figure majeure de l'histoire de la médecine sous la Révolution française, et plus particulièrement encore sous le Directoire. Médecin-chef de l'hôpital de Bicêtre puis de la Salpêtrière à partir de 1795, auteur d'une Nosographie philosophique en 1798 puis d'un Traité médicophilosophique sur l'aliénation mentale en $1801^{7}$, il s'impose dans l'espace médical et scientifique, un succès que couronne son élection à l'Académie des sciences en $1803^{8}$. La réputation indéniable dont il est l'objet doit être précisément replacée dans le contexte particulier de la République directoriale, de l'invention de la Terreur et de la relation particulière (re) construite en l'an III entre les élites républicaines et le peuple.

(7) Philippe PINEL, Traité médico-philosophique sur l'aliénation mentale ou la manie, Paris, chez Richard, an IX.

(8) Sur Philippe Pinel, la bibliographie est importante. Signalons : Jacques Postel, Genèse de la psychiatrie, Le Sycomore, 1981 ; Jean GARRABÉ (dir.), Philippe Pinel, Paris, Les Empêcheurs de tourner en rond, 1994 ; Jan GOLDSTEIN, Consoler et classifier. L'essor de la psychiatrie française, Paris, Les Empêcheurs de penser en rond, 1997 ; Dora W. WeINER, Comprendre et soigner. Philippe Pinel (1745-1826). La médecine de l'esprit, Paris, Fayard, 1999 ; Jackie PIGEAUd, Aux portes de la psychiatrie. Pinel, l'ancien et le moderne, Paris, Aubier, 2001. 


\section{La folie, une maladie politique}

La légitimation de l'élimination suivie de l'exécution de Robespierre comme la construction progressive d'une interprétation visant à réduire la Terreur à un régime assimilé aux violences, aux dérèglements aboutissent à mettre la folie à l'ordre du jour. Les portraits de Robespierre qui suivent le 9 Thermidor insistent sur la «mélancolie » dont aurait souffert le désormais tyran. Dans un discours de circonstance prononcé dans les jours qui suivent la chute de Robespierre, le député Merlin de Thionville met au jour les ressorts psychologiques d'un tyran ravalé au statut de malade :

«Le tempérament de Robespierre fut d'abord mélancolique, il finit par être atrabilaire. À l'Assemblée constituante, il avait le teint pâle ; à la Convention, il devint jaune et livide ; longtemps, il ne parla à l'Assemblée constituante qu'en gémissant; à la Convention, il ne parlait qu'en écumant. [...] À la suite, l'atrabile a fait du mouvement de ses idées, une tourmente ; de ses idées d'effroyables fantômes ; de son imagination, une furie ${ }^{9}$.

Ces portraits, repris et diffusés dans la presse, sur les théâtres et dans les premiers mémoires politiques de l'an $\mathrm{III}^{10}$, fixent pour longtemps les fondements de la représentation de Robespierre et des quelques autres figures sur lesquelles se cristallisent alors l'abjection politique ${ }^{11}$. De ces quelques cas individuels, la folie se diffuse progressivement au peuple et se découvre particulièrement lors des révoltes parisiennes du printemps 1795. Cause explicative des comportements déviants, la maladie mentale constitue alors un moyen de stigmatiser le « mauvais » peuple et permet d'expliquer toutes les violences comme l'assassinat du représentant Ferraud. Le romancier et publiciste girondin Jean-Baptiste Louvet s'érige en porte-parole des élites thermidoriennes en mettant en scène de manière particulièrement frappante la folie du peuple :

«À la tête de quelques braves, Ferraud se précipite; et quel spectacle a frappé ses yeux ! C'est la déraison, l'imposture, la colère, l'impudeur, l'impudence ; ce sont les vengeances, les haines, les viles imprécations, les

(9) Merlin de Thionville, représentant du peuple à ses collègues, portrait de Robespierre, Paris, 1794, p. 1-2.

(10) Par exemple, Dominique GaRat, Mémoires sur la Révolution ou exposé de ma conduite dans les affaires et dans les fonctions publiques, Paris, an III/1795, p. 24.

(11) Antoine de BAECQUE, La gloire et l'effroi. Sept mort sous la Terreur, Grasset, 1997 ; Guillaume MAZEAU, Le bain de l'histoire, Seyssel, Champ Vallon, 2009. 
malédictions féroces, toutes les passions hideuses, toutes les fureurs, toutes les furies $»^{12}$.

Le discours thermidorien ne retient de ces journées que le déchaînement de la violence et vide la révolte de toute signification politique : le soulèvement populaire apparait dès lors comme le signe de la folie ${ }^{13}$. Dans ce contexte, il devient urgent de rétablir l'ordre de la Raison. La République ne saurait dès lors échapper aux hommes raisonnables, aux propriétaires et aux savants ${ }^{14}$. Si tous les hommes sont doués de raison, seuls ceux qui savent «bien » penser, selon les règles de l'analyse, de l'observation, rappelées avec force au sein même de la Convention nationale, méritent d'être aux commandes politiques. Condillac et ses théories sensualistes s'imposent alors comme une référence incontournable pour les élites thermidoriennes. C'est dans ce contexte particulier qu'il faut replacer la valorisation de Pinel et de ses travaux. Son traitement moral, qui consiste à rechercher les moyens de rétablir la raison que n'abolit jamais la folie considérée comme un état de dérèglement plus ou moins passager, répond donc à une volonté d'inclusion. Il constitue le symbole de la vaste entreprise de « républicanisation » des esprits, projet d'une thérapie politique dans laquelle les républicains du Directoire sont engagés pour mieux affirmer la rupture avec la $«$ Terreur $»^{15}$.

Dès 1796, Pinel est ainsi érigé en sauveur de la République. Ses travaux sur les dysfonctionnements de la Raison et sur les moyens d'en prévenir les «excès » prennent une importance nouvelle et justifient la valorisation de ce médecin comme figure majeure du «sacerdorce » médical. Selon les fondements épistémologiques des rapports du physique et du moral théorisés en 1796 par Pierre-Jean-Georges Cabanis, Pinel légitime l'intervention du savant sur le terrain du «moral » et de la morale. Les récits des différentes causes des maladies qu'il présente dans son Traité

(12) «Éloge de Ferraud par Louvet à la Convention nationale », Moniteur universel, séance du 14 prairial an III, vol. 24, p. 608.

(13) Raymonde MONNIER, «Le tournant de Brumaire : dépopulariser la révolution parisienne ", dans Michel Vovelle (dir.), Le tournant de l'an III. Réaction et Terreur blanche dans la France révolutionnaire, Paris, Éditions du CTHS, 1997, p. 187-199.

(14) Jean-Luc CHAPPEY, « Raison et citoyenneté : les fondements culturels d'une distinction sociale et politique sous le Directoire », dans Raymonde MONNIER (dir.), Citoyen et citoyenneté sous la Révolution française, Actes du Colloque de Vizille du 24-25 septembre 2005, Paris, Société des études robespierristes, 2006, p. 279-288.

(15) Cette réflexion doit beaucoup aux discussions menées avec Anne Simonin, Pierre Serna, Clyde Plumauzille et Virginie Martin. 
médico-philosophique peuvent être lus comme autant de contributions à une histoire immédiate de la Révolution :

«Un citoyen estimable, ayant perdu sa fortune par des événements de la révolution, fut bientôt conduit dans un état de tristesse profonde à un état de manie bien caractérisé [...]. Un jeune consterné du renversement du culte catholique en France, et dominé par des préjugés religieux devint maniaque. [...]. Un fameux horloger de Paris perdit la raison par le double effet de la chimère du mouvement perpétuel qui avait égaré son imagination, et par les terreurs sans cesse renaissantes qu'excitaient les orages de la révolution. [...]. Un ouvrier, réduit à l'état d'aliénation par suite des terreurs excitées à une des époques les plus orageuses de la révolution... $»^{16}$.

Selon Antoine Destutt de Tracy, membre de la Classe des sciences morales et politiques de l'Institut national et porte-parole du courant des Idéologues, « en expliquant comment les fous déraisonnent, [Pinel] apprend aux sages comment ils pensent [...]. Ce sont les physiologistes philosophes comme le citoyen Pinel qui avanceront l'Idéologie $»^{17}$. Présentée alors comme la science dont la construction doit renforcer la stabilité de la République, l'Idéologie doit ainsi prévenir toute possibilité d'un retour à la « barbarie » en évitant les nouveaux risques de crises de folie politique. Dans ce contexte, l'importance de la mission assignée aux « médecins » et aux savants, incarnés en particulier par la figure de Pinel, se mesure à la violence des attaques dont ils sont l'objet de la part des publicistes catholiques et des adversaires de la République mise en place en l'an III. Alors que le débat sur la folie dépasse largement les limites du terrain médical ou administratif, les autorités politiques décident de rouvrir l'hospice de Charenton afin de dégorger les hôpitaux de la Cité, de la Salpêtrière et de Bicêtre.

\section{Coulmier, directeur de Charenton}

Située aux environs de Paris, la Maison de Charenton est ouverte en 1641 par une donation aux frères de la Charitée ${ }^{18}$. Elle accueille au

(16) Jacques-Louis MOREAU DE LA SARTHE, « Traité médico-philosophique sur l'aliénation mentale par Ph. Pinel », Décade philosophique, section Art de guérir, 29e vol., (mars-mai 1801), $3^{\mathrm{e}}$ trim., p. 458-467.

(17) Cit. dans François PICAVET, Les idéologues. Essai sur l'histoire des idées et des théories scientifiques, philosophiques, religieuses en France depuis 1789, Paris, F. Alcan, 1891, p. 174.

(18) Marie-Geneviève RICHARD, L'histoire oubliée de Charenton-Saint-Maurice, SaintMaurice, chez l'auteur, 1972 ; Pierre PINON, L'hospice de Charenton, Liège, Mardaga, 1989. 
XVIII ${ }^{\mathrm{e}}$ siècle des « marginaux », pauvres, aliénés, mais aussi des prisonniers par lettres de cachet, justifiant ainsi les nombreuses dénonciations dont elle devient l'objet ${ }^{19}$. Elle est fermée le 12 messidor an III (30 juin 1795) par le Comité des secours publics pour être utilisée comme prison pour les militaires du camp de Vincennes, militaires qui resteront présents dans la Maison nationale de Charenton qui recevra de nombreux officiers envoyés de l'hôtel des Invalides. Face aux difficultés d'accueil que rencontre l'hôpital de l'Hôtel-Dieu, le Directoire exécutif prend la décision, le 27 prairial an V(15 juin 1797), d'ouvrir la Maison nationale de Charenton. Sur les recommandations de Michel-Augustin Thouret (1748-1810), directeur de l'École de médecine de Paris, le ministre de l'Intérieur nomme François-Simonet Coulmier à la fonction de régisseur-général de l'hôpital de Charenton. Il entre en fonction le $1^{\text {er }}$ vendémiaire an VI (22 septembre 1797), dans un contexte politique particulièrement agité ${ }^{20}$.

L'établissement est placé directement sous l'autorité du ministre de l'Intérieur (qui nomme son directeur et son personnel) et soumis au même statut que deux autres institutions importantes, l'Institut national des sourds et muets dirigé par l'abbé Sicard (1742-1822) et l'Établissement national des aveugles dirigé par Valentin Hauÿ (1845-1822) ${ }^{21}$. Charenton reste donc un «établissement de bienfaisance » soumis à un régime spécial caractérisé par les pouvoirs importants accordés au Directeur qui n'est pas soumis à la surveillance du corps des médecins. En 1798, il est prévu que les professeurs de l'École de médecine sont « chargés de l'inspection de la maison des insensés de Charenton pour tout ce qui intéresse le régime médical et le traitement des individus » et de «présenter au ministre de l'Intérieur les règlements propres à régulariser ce service $\gg^{22}$. Le reste des charges relève des seules décisions du directeur. Comment justifier la nomination de Coulmier à la tête de cet établissement ?

Les difficultés financières auxquelles sont confrontées les autorités du Directoire et la nécessité de confier l'établissement à un gestionnaire compétent justifient sans doute la recommandation de Thouret dont le frère, Guillaume (1746-1794), avocat, député de la Constituante,

(19) Gabriel VAUTHIER, « La maison de Charenton en 1790 », AHRF, 1926, tome III, p. 264-274 ; Jacques Postel, «Une visite des Commissaires de la Mairie de Paris à la Maison de Charenton en décembre 1790 », L'Évolution Psychiatrique, 49, 1, 1984, p. 257-262.

(20) Sa nomination suit de près le coup d'État républicain du 18 fructidor an V(5 septembre 1797) contre les « royalistes ».

(21) Jean-Luc ChapPey, La Société des Observateurs de l'homme. Des anthropologues sous Bonaparte, Paris, Société des études robespierristes, 2002 ; François BUTON, L'administration des faveurs. L'État, les sourds et les aveugles (1789-1885), Rennes, PUR, 2009.

(22) AN, AJ16 6226, Fol. 313-314. 
côtoyait Coulmier au Comité de mendicité. Fils d'une famille anoblie au XVIII ${ }^{\mathrm{e}}$ siècle par la charge de secrétaire du roi à la grande chancellerie de France $^{23}$, ce dernier commence une carrière ecclésiastique. Il entre dans les ordres en 1764, chanoine à l'abbaye des Prémontrés de Chambrefontaine et prieur-curé de Congis. Visiblement réputé pour ses qualités d'administrateur, il devient, en 1783, abbé régulier de Notre-Dame d'Abbécourt, toujours rattaché à l'ordre des Prémontrés ${ }^{24}$. Il relève les finances d'une maison endettée, encadre lui-même un important programme de rénovation des bâtiments et développe les cultures vivrières et l'élevage. En 1789, il est élu député du clergé par les électeurs de Paris Hors-les-Murs. Il soutient alors la réunion du Clergé au Tiers ${ }^{25}$ et devient successivement membre de quatre comités de la Constituante entre 1789 et $1790^{26}$. Il participe activement aux débats sur la Constitution civile du Clergé à laquelle il prête serment et intervient plus particulièrement dans les débats touchant aux questions économiques comme l'illustre son intervention en faveur de la défense de la culture du tabac en $1790^{27}$. Il participe aux réunions de la Société nationale des Neuf Sœurs au sein de laquelle il prononce le 14 janvier 1791 un discours pour justifier son serment en faveur de la Constitution civile. Il se met en retrait des affaires publiques à partir de 1792, mais est inquiété en 1793 du fait de l'émigration de sa nièce. Il décide de quitter la France alors que son frère, Claude François, est exécuté en 1794 comme fermier-général. Il rentre au lendemain du 9 Thermidor et participe à la gestion d'une ancienne propriété appartenant aux Lazaristes achetée, près de Sevran, comme bien national. Les autorités voient en Coulmier sans doute plus un gestionnaire qu'un spécialiste en traitement

(23) Son père, Jacques, écuyer seigneur d'Escolmiers, est Trésorier général de France à Dijon (1732-1757), puis secrétaire du roi à la grande chancellerie (1732-1757). Sa mère, Anne Rougeot, est la fille d'un Receveur général des domaines et bois en Bourgogne et Bresse, sœur de fermier général. Sa tante, Anne Simonnet, épouse en 1709 François Morel, négociant banquier à Lyon puis à Paris, secrétaire du roi à la grande chancellerie et conseiller à la monnaie de Lyon. Edna H. LEMAY, Dictionnaire des Constituants 1789-1791, Paris, Universitas, 1991, p. 240

(24) Cette position lui rapporte une rente de 6000 livres. Cf. Pierre-Yves LouIs, « Monsieur de Coulmier : François-Simonet des Coulmier (1741-1818), dernier abbé régulier de l'assemblée nationale constituante et premier directeur de la maison nationale de Charenton », Actes officiels du $15^{e}$ colloque du centre d'études et de recherches prémontrés, Mont-Saint-Odile, 1989, p. 40-51.

(25) Il publie par exemple une Motion sur l'urgence de la réunion des trois états, Paris, 1789. Il plaide en novembre 1789 pour que la mise à disposition de la nation des biens du clergé ne soit pas réalisée de manière humiliante. En décembre, il prend position en faveur d'une augmentation du niveau de vie des curés. En novembre 1790, il intervient sur la retraite du clergé régulier et sur le statut des nouveaux évêques.

(26) Il appartient successivement au comité de Vérification (22 juin 1789), de Subsistances (25 juin 1789), de Mendicité (21 janvier 1790) et d'Aliénation des Domaines nationaux (17 mars 1790).

(27) Opinion sur le serment, Paris, janvier 1791 par M. l'abbé d'Abbécourt (De Coulmier), député à l'Assemblée nationale, Paris, de l'imprimerie de la Société nationale des Neuf-Sœurs, [1791]. 
de la folie. Nous verrons justement qu'il parvient à faire de Charenton un laboratoire du traitement moral entre 1797 et 1806.

\section{Charenton, un laboratoire réputé dans le traitement moral}

Dès 1797 , la maison accueille plusieurs types de malades qui sont logés, installés et entretenus dans des conditions très différentes les uns des autres. Les différents registres font apparaître trois grandes catégories de malades, mêlant hommes et femmes, riches et pauvres : la plus nombreuse, nous l'avons déjà évoquée, est celle des militaires invalides, aliénés et souvent placés par leurs autorités ; les militaires qui ne sont pas invalides côtoient les pensionnaires civils qui sont à leur tour distribués, selon leurs revenus, en trois catégories. Des listes envoyées par la Commission des hospices et de l'Hôtel-Dieu de Paris permettent de mieux apprécier l'évolution du nombre de cette dernière catégorie de malades civils. S'il est difficile de mesurer exactement le nombre des pensionnaires, celui-ci semble avoir progressé régulièrement entre 1797 et 1805 : de 98 pensionnaires en 1802, le nombre des patients envoyés par les autorités administratives passe à 132 en 1803 , à 177 en 1806 et à 60 en $1808^{28}$. Ces variations peuvent, en partie, dépendre des envois, par la préfecture de Police de Paris et la Commission sénatoriale des libertés établie le 6 juin 1804, de certains prisonniers considérés comme « fous », susceptibles de sortir quelque temps après. Selon Michel Vovelle, c'est dans cette logique répressive que Théodore Desorgues est envoyé à Charenton en mai 1805, l'établissement jouant ainsi le rôle de «prison politique $»^{29}$. La plupart de ces opposants présentés comme fous par les autorités prennent à leur charge les frais de leur séjour. Ces malades vivent dans un vaste établissement composé de plusieurs parties et offrant des services étendus, adaptés aux différents traitements nécessaires. En bon gestionnaire, Coulmier y développe des activités variées (cultures, jardinage, écuries) permettant à l'institution de se fournir en produits, certains malades travaillant pour l'économie de la maison, d'autres, plus aisés, pouvant bénéficier d'un certain confort. Les enjeux de ces aménagements ne se réduisent pas à l'économie. Selon les principes du «traitement moral » défendus par Coulmier, soigner la folie consiste à agir sur le milieu et l'environnement du malade : favoriser les promenades et les relations humaines, occuper et

(28) Anne-Marie BERNARD et Jacques HoudAILLE, « Les internés de Charenton, 1800-1864 », Population, 1994, vol. 49, n², p. 500-515.

(29) Michel VovelLE, Théodore Desorgues ou la désorganisation. Aix-Paris, 1763-1808, Paris, Seuil, 1985, p. 198 et sq. L'auteur présente plusieurs cas d'incarcération « par mesure de sûreté ». 
divertir les malades en améliorant ses conditions de vie doivent participer à l'œuvre thérapeutique offerte dans l'hôpital de Charenton.

Dès sa prise de fonction, Coulmier doit faire œuvre de tout bois pour maintenir à flot les finances de l'institution tout en veillant à l'accueil des malades. Il s'attèle ainsi à recouvrer les sommes dues par les institutions ou les familles qui ont placé des malades, à récupérer également des loyers versés sur les terres ou les habitations louées par l'hôpital. Il s'occupe encore de l'entretien des bâtiments et, comme à Abbécourt, il en fait également construire de nouveaux. Entre 1797 et 1814, face à l'afflux des malades, la surface bâtie triple de volume grâce à de nouvelles constructions ou à des annexions de bâtiments. Une lettre du 10 janvier 1798 au citoyen Molinot, chef de la $2^{\mathrm{e}}$ division du ministère de l'Intérieur le montre ainsi employé à des entreprises de récupération de terres : Coulmier informe son autorité de tutelle qu'il a demandé à son ancien collègue, le ministre des Finances, de récupérer quelques « dépouilles » venant des biens des princes de la maison de Bourbon. Il s'appuie sur une loi de septembre 1797 permettant aux institutions nationales de récupérer certains des biens vendus au titre de biens nationaux. En dépit de ses efforts, Coulmier doit souvent, comme le montre cette lettre du 30 décembre 1802 au ministre de l'Intérieur, solliciter le soutien financier des autorités. L'institution semble alors souffrir de son succès qui a pour effet l'augmentation du nombre de malades dont l'hétérogénéité sociale n'est pas sans poser problèmes, le directeur devant encore s'occuper des problèmes qui peuvent naître du voisinage entre riches et pauvres au sein de son institution.

Coulmier n'est évidemment pas le seul à administrer l'établissement. Le personnel soignant passe de dix-huit membres en 1798, à trente en 1802 pour atteindre soixante en 1810 . Coulmier s'est entouré de proches comme le chirurgien Deguise, qui réside à côté de l'hospice ou le deuxième surveillant général, Dumoustier, ancien prieur d'Abbécourt. Jean-Baptiste Joseph Gastaldy (1741-1805), nommé médecin-chef en décembre 1797, occupe une place importante parmi le personnel. Docteur de la Faculté de Montpellier, centre important des réformes médicales au XVIII ${ }^{\mathrm{e}}$, particulièrement dans le domaine des nosologies et des recherches sur les rapports du physique et du moral, Gastaldy devient, en 1767, médecin chef de l'hôpital Sainte-Marthe d'Avignon. Participant à différentes campagnes menées contre diverses épidémies, il est correspondant de la Société royale de médecine et membre de la Loge de la Parfaite Union. En 1790, il quitte le Comtat pour s'établir à Paris puis décide de rejoindre l'Angleterre où il devient médecin particulier du duc de Cumberland, Henry Frederick (17451790), le jeune frère du roi George III. Rentré sous le Directoire, il se voit 
de nouveau confier une série de missions contre les épidémies puis entre à Charenton où il joue, en collaboration avec Coulmier, un rôle essentiel dans la mise en pratique du traitement moral de Pinel. Les visites que rendent les autorités administratives témoignent par ailleurs de la satisfaction de ces dernières. Présenté le 4 floréal an XII au ministre de l'Intérieur, Chaptal, le Mémoire sur la Maison nationale de Charenton, exclusivement destinée au traitement des aliénés rédigé par le médecin Charles-François-Simon Giraudy (1770-1848), autre médecin réputé ${ }^{30}$, collaborateur de Gastaldy, offre un tableau précis, indéniablement laudateur, de l'administration et des traitements offerts aux malades de Charenton :

«La maison nationale de Charenton est la première en France qui ait été uniquement destinée au traitement des aliénés ; la première où la présence des incurables ne nuise point à ceux qui sont encore susceptibles de guérison; où les malades ne soient pas considérés, dans tous les cas, comme totalement dépourvus de jugement; où ils ne soient pas exposés à des brutalités, ni à des mauvaises plaisanteries, ni à tous les autres traitements inhumains $»^{31}$.

Selon la nature de la maladie mentale, les traitements apportés aux patients mêlent en effet des formes contraignantes consistant à agir sur le « physique » (douches, bains, enfermement...) et des formes plus « douces » visant à rétablir la relation sociale avec le malade et à le réintégrer dans un espace de communication. De visite à Paris, le médecin allemand August-Friedrich Schweigger signale particulièrement le rôle joué par les représentations théâtrales dans les pratiques thérapeutiques ${ }^{32}$. Fondé sur les principes épistémologiques des rapports du physique et du moral, le traitement moral vise en effet à faire sortir l'aliéné de son isolement. Dès lors, il convient autant d'agir sur les corps (parfois de manière violente) que sur le moral en utilisant des méthodes qui doivent rendre possible ce « dialogue avec l'insensé », justifiant l'importance accordée aux différentes formes de parole, communication orale, mais aussi danse, musique ou théâtre, autant d'outils thérapeutiques utilisés par Pinel à Bicêtre mais

(30) Il est directeur du Journal de médecine pratique (1806-1819).

(31) Charles-François-Simon GIRAUDY, Mémoire sur la Maison nationale de Charenton, exclusivement destinée au traitement des aliénés, Paris, impr. de la Société de médecine, an XII/1804, Avis.

(32) « Une visite des établissements d'aliénés parisiens en 1808 », par August-Friedrich SCHWEIGGER, traduit de l'allemand par Michel Caire et mis en ligne http://psychiatrie.histoire.free.fr/ index.htm 
aussi par l'abbé Sicard dans son établissement pour les sourds ${ }^{33}$. Sur ce point, il semble incontestable que Coulmier ait tenté de développer, en leur donnant plus de portée, certaines des expériences menées dans d'autres établissements. Si les archives ne permettent pas de préciser la nature des spectacles, il apparaît que, sur ce point, Charenton constitue un lieu d'expériences pour des pratiques souvent redécouvertes au $\mathrm{XX}^{\mathrm{e}}$ siècle ${ }^{34}$. Comme le rappelle cet extrait d'un « Avis » vantant les ressources offertes par l'hôpital, les malades disposent, selon leur richesse, de logements et de services différents :

« Il y a des chambres communes, des chambres particulières à cheminées et des appartements composés de plusieurs pièces pour ceux à qui leur fortune permet la facilité d'avoir un domestique particulier. Des terrasses du grand jardin sont destinées à la promenade ; on conduit dans la campagne les malades dont l'état permet de sortir au-dehors. Il y a des salles d'assemblées, de billard et de jeux tels que les dames, les échecs, le trictrac, et une bibliothèque ; enfin, on y réunit tous les genres d'amusement et de distraction : car l'isolement est un moyen d'augmenter la folie, tandis que la société et la vie commune en est un de l'adoucir. La table y est bonne, cette jouissance étant souvent la seule qu'on puisse procurer dans cet état : la nourriture consiste en potages, viandes et volailles bouillies et rôties, poisson, pâtisseries, légumes et fruits. Le prix du pensionnat diffère à raison de l'aisance et des jouissances que la fortune de l'individu permet à sa famille de lui procurer : le minimum est de six cents francs par an. La famille fournira le coucher, quatre paires de draps, deux douzaines de serviettes, et se chargera de l'habillement : la maison pourra procurer ces objets moyennant une somme additionnelle dont on conviendra $»^{35}$.

Même si aucune source ne permet d'en connaitre la nature, l'organisation de spectacles mettant en scène des malades constitue un moyen susceptible de favoriser leur guérison et entre dans une stratégie publicitaire indéniable. Pour le directeur, toujours soucieux de trouver des ressources, l'organisation de ces spectacles sert aussi à attirer un public de curieux, venus pour des raisons plus ou moins avouables, qui paie pour assister à ces représentations. Même si les archives à disposition ne permettent

(33) Gladys SwAIN, Dialogue avec l'insensé, Paris, Gallimard, 1993 ; Laurence BROCKLISS, Colin JonES, The Medical World of the Early Modern France, Oxford, Clarendon Press, 1997.

(34) Cf. le film de 1996 de Nicolas PHILIBERT, La moindre des choses : http://www.youtube. com/watch?v=Ox9nWVSYvTU

(35) « Avis », Paris, de l'imprimerie de la République, nivôse an VI. 
pas d'en mesurer la place exacte, il semble que Charenton se soit ainsi imposé dans la géographie des théâtres parisiens, particulièrement à partir de l'entrée comme «pensionnaire » du fameux marquis Donatien de Sade (1740-1814), le 7 floréal an XI (27 avril 1803) ${ }^{36}$. Ce dernier a sans doute bénéficié d'une initiative lancée avant lui et l'on peut croire que, sous sa direction, les spectacles proposés ont bénéficié d'une portée plus grande.

De manière générale, il semble que le personnel de Charenton soit parvenu à imposer la réputation de l'établissement comme espace thérapeutique où les conditions de vie des patients ne sont pas négligées. En 1804, le médecin adjoint, Charles-François Giraudy, qui vise indéniablement à valoriser l'établissement, signale que la nourriture est « saine et abondante [...] Elle est la même pour l'indigent et pour le riche ; et là l'observateur voit avec plaisir que le mot Égalité n'est pas un mot vide de sens. Un potage, un bouilli, une entrée et un dessert forment le dîner ; un rôti, un entremets compose le souper; et l'on donne à chaque repas une quantité de vin proportionné à l'état de chaque malade ${ }^{37}$. Soucieux encore de promouvoir les efforts du personnel pour améliorer les conditions de vie des malades, il décrit encore dans le Journal général de médecine, une « gangue de force » qui devait remplacer la camisole de force afin de diminuer les souffrances liées aux entraves ${ }^{38}$. Durant son administration, Coulmier se montre d'ailleurs curieux des possibles découvertes thérapeutiques dans le domaine du traitement de la folie. En 1807, il écrit par exemple à un de ses confrères, Pierre-Antoine Prost (1770-1832), directeur depuis 1805 d'une Maison de santé située à Montmartre ( $a \mathrm{n}^{\circ} 4$ de la rue Trainée, actuelle rue Norvins), qui, dans son ouvrage Coup d'œeil physiologique sur la folie ${ }^{39}$,

(36) Il existe une littérature très abondante concernant la présence de Sade à Charenton. Signalons, Isabelle Tatiana REDALIE, L'internement de Sade à Charenton. Considérations sur les deux internements de Sade à l'hospice de Charenton, leur signification historique et médico-légale, Thèse de doctorat en médecine, Paris, 1969.

(37) Charles-François GIRAUDY, Mémoire sur la Maison nationale de Charenton, Imprimerie de la Société de médecine, 1804 , p. 13.

(38) «Sur un nouveau moyen de contenir les aliénés furieux, employé à l'hospice national de Charenton ; avec quelques réflexions sur ceux des moyens connus qui ont obtenu la préférence jusqu'à ce jour, par M. Giraudy, médecin », Journal général de médecine, de chirurgie et de pharmacie, t. 19, an XII/1803, p. 148-152.

(39) Pierre-Antoine Prost, Coup d'œil physiologique sur la Folie ou Réflexions et recherches analytiques sur les causes qui disposent à cette maladie, et sur celles qui lui donnent lieu et qui l'entretiennent; suivies des diverses méthodes qu'il faut employer dans son traitement en raison de ces causes. ce sujet est traité sous des points de vue nouveaux : c'est une exposition succincte de faits déjà connus, et de faits découverts depuis peu sur les cadavres, particulièrement dans les viscères du ventre où l'on trouve les causes essentielles de cette maladie, Paris, Chez Demonville et chez l'Auteur, s.d. [1806]. 
propose de nouvelles hypothèses sur les causes des malades mentales ${ }^{40}$. Plusieurs témoignages postérieurs rappellent en outre que l'établissement était largement ouvert sur la ville de Charenton, les patients, du moins ceux qui en avaient la possibilité, étant libres de se promener hors des limites de l'hospice, pouvant même se rendre à Paris ${ }^{41}$. Sous l'administration de Coulmier, l'hôpital semble bénéficier d'une aussi bonne réputation que les établissements parisiens, les autorités administratives et les particuliers n'hésitant pas à y envoyer les patients souffrant, à des degrés divers, d'une folie réelle ou supposée. L'établissement accueille ainsi le marquis de Sade, le chansonnier Théodore Desorgues ou le conventionnel, député d'Indre-et-Loire, Jacob Louis Dupont (1755-1813) à partir de 1798. La bonne réputation de son établissement entre sans doute en compte dans l'élection de Coulmier autant au Corps législatif en 1799 que dans diverses institutions locales : il est président du conseil électoral de Sceaux puis du canton de Charenton. Il inspire même quelques auteurs de pièces de théâtre, tel Joseph Berchoux, auteur du Philosophe de Charenton en $1803^{42}$. Or, les choses vont se dégrader assez rapidement. En 1805, Gastaldy décède. Son remplacement va être à l'origine d'un conflit particulièrement violent aboutissant à la perte de Coulmier.

\section{Charenton, théâtre de la cruauté ?}

À la suite du décès de Gastaldy, plusieurs réseaux d'influences et de solidarités se mobilisent pour peser sur le choix du successeur auprès du ministre de l'Intérieur, Champagny. Coulmier transmet à ce dernier une

(40) La thèse de Prost est que les vers intestinaux sont la cause la plus fréquente de l'aliénation mentale. L'existence de manies vermineuses est défendue également par le jeune Esquirol. Cette thèse est attaquée par Royer-Collard dans la Bibliothèque médicale Bibliographie médicale ou Recueil périodique d'extraits des meilleurs ouvrages de médecine et de chirurgie par une société de médecins, Paris, Vinçard, 1807, t. XV, p. 133-136. Prost répond, dans son Troisième coup d'œil physiologique sur la folie

(41) Jean-Étienne ESQUIROL, Mémoire historique et statistique sur la maison royale de Charenton, Paris, Paul Renouard, 1835.

(42) Cf. Le philosophe de Charenton, Paris, Giguet et Michaud, an XI/1803. 
« fiche de poste » qui détaille les contraintes de la fonction ${ }^{43}$. Il en profite pour proposer une liste de quelques noms :

« Je crois qu'il est d'une nécessité pour ainsi dire indispensable que la personne que vous aurez la bonté de choisir réside à Charenton. [...] Ce qui me détermine à proposer pour un des candidats $\mathrm{M}$. Deguise, nommé en l'an 4, chirurgien de l'hôpital de Charenton, qui depuis cette époque et conformément aux nouveaux règlements, a été nommé docteur en médecine [...] M. Sédillot, médecin très estimable, sollicite la place. Il est protégé par M. le sénateur Lacepède, grand chancelier de la Légion d'Honneur, par M. [Baviere], sénateur, recommandations respectables et infiniment honorables pour M. Sédillot. Mais il est très occupé sur la place de Paris. Secrétaire de la Société de médecine, je craindrais que ces grandes occupations ne lui permissent pas de donner aux malades tous les soins qu'exige leur pénible situation $»^{44}$.

D'autres personnalités cherchent alors à placer leur protégé, Pinel faisant alors campagne en faveur de son élève Jean-Étienne Esquirol (1772-1840). Or la décision est prise le 23 janvier 1806, en partie par les membres d'une commission composée de Joseph-Marie de Gérando, Mathieu de Montmorency et Barbier-Neuville qui décident de nommer Antoine-Athanase Royer-Collard. Ce dernier est «protégé et lié avec M. Portalis, secrétaire général du ministère du Culte ; et quelque temps avant la disgrâce de ce dernier, il était sur le point d'être nommé par lui, inspecteur général de la librairie ». Il est aussi frère du député Pierre Paul Royer Collard, professeur d'humanités, dans la congrégation libre de l'Oratoire, il est employé pendant quelque temps à l'administration des vivres des Alpes, emploi qu'il abandonne en $1797^{45}$. Après avoir soutenu une thèse

(43) « 1- La visite du médecin se fera tous les jours en été à six heures du matin, et à huit heures en hiver. Le médecin sera toujours accompagné d'un surveillant, d'un des élèves en chirurgie et du garçon de pharmacie qui tiendra le registre des ordonnances. Cet article est d'autant plus intéressant que les malades resteront dans leurs chambres jusqu'après la visite. C'est le seul moyen pour que le médecin puisse juger dans le calme de l'effet des remèdes qu'il a ordonné. La visite étant faite à cette heure, les malades pourront jouir de la liberté de la promenade avant le moment de la grande chaleur, mesures que l'usage et l'expérience me font regarder comme indispensables. 2- Le médecin sera présent aux douches qu'il aura ordonnées au moins une fois par semaine afin de pouvoir juger de leur effet. 3- Le médecin fera tous les ans, conjointement avec le directeur général, un rapport à votre excellence sur le nombre des malades, des traitements pendant l'année, des cures qui se seront faites, avec des observations sur les causes présumées de la maladie » [AD Val-de-Marne, AJ2 100]

(44) A D Val-de-Marne, 2 Mi 56, « Rapport au ministre de l'Intérieur sur la nomination d'un médecin » [1804].

(45) Il collabore à la rédaction du Surveillant, par une société de patriotes, Lyon, 1791. 
de doctorat en $1802^{46}$, il devient rédacteur de la Bibliothèque médicale en $1803^{47}$ et participe à la légitimation de la loi du 19 ventôse an XI de réorganisation de la profession médicale. Il n'a pas forcément une très bonne réputation au sein du monde médical. Dès sa prise de fonction à Charenton, Royer-Collard est sollicité comme « expert » par les tribunaux de la Seine dans plusieurs affaires familiales pour mesurer la maladie d'individus placés en tutelle par leur famille. Mis en cause dans l'une d'entre elles, Royer-Collard doit se défendre en publiant en décembre 1806 des Observations ${ }^{48}$ où il s'en prend particulièrement à Jean-Baptiste Pussin (1745-1811), le fameux auxiliaire de Pinel.

Entre 1806 et 1814, Coulmier et Royer-Collard vont s'affronter de manière de plus en plus violente, chacun n'hésitant pas à user de tous les moyens pour s'attaquer à la réputation de son adversaire ${ }^{49}$. Dans une lettre envoyée le 29 janvier $1806^{50}$, Royer-Collard informe Coulmier de sa nomination à la place de médecin chef et s'enquiert auprès du médecin Deguise de l'état des malades. Le nouveau médecin souhaite recevoir les registres d'entrée et de sortie des malades, registres tenus par Coulmier et relevant de prérogatives administratives. Deguise l'informe par ailleurs de la difficulté à obtenir certains renseignements sur l'état des malades et la cause de leur maladie :

«M. de Coulmier à qui j'ai fait part du désir que vous aviez de composer la liste des malades actuellement en traitement à Charenton m'a dit qu'il s'occuperait de ce travail aussitôt que, conformément à la volonté du ministre de l'Intérieur, il aurait évacué les malades de l'hôtel-dieu et de la police qui ont plus de six mois de résidence [...] Vous demandez ensuite, Monsieur, des détails qui ne sont jamais parvenus à la maison

(46) Essai sur l'aménorrhée ou suppression du flux menstruel, Paris, 26 thermidor an 10.

(47) Bibliothèque médicale ou Recueil périodique d'extraits des meilleurs ouvrages de médecine et chirurgie par une société de médecins..., Paris, Gabon \& Méquignon-Marvis, 1803-1822, 78 vol.

(48) Observations sur un récit ayant pour titre "Mémoire pour Mme de Chabon, appelante du jugement qui nomme M. Fréteau administrateur provisoire de la personne de d'Abbouville; contre M. Dupaty et M. et Mme de la Chatagnerai, intimés » par M. Royer-Collard, docteur en médecine, membre de la Société de l'École de médecine de Paris, médecin en chef de l'hospice de Charenton et l'un des médecins chargés par le tribunal de première instance du département de la Seine, du traitement de Mlle d'Abbouville, Paris, de l'imprimerie de Vinçard, décembre 1806.

(49) Un dossier conservé aux Archives nationales [AJ2 100] porte pour titre « Démêlés entre M. de Coulmier et le S. Royer-Collard, 1806-1813 ». Il comporte douze pièces et a déjà fait l'objet de plusieurs études et donné lieu à des interprétations. Cf. Jan GOLDSTEIN, Console and Classify : The French Psychiatric Profession in the Nineteenth Century, University of Chicago Press, 2002. $56]$.

(50) « Lettre de Royer-Collard », 29 janvier 1806 [AN, AJ2 100/ AD Val-de-Marne, 2 Mi 
vu la différente classe des malades qui y viennent de la police de l'Hôteldieu, des Invalides. Messieurs Coulmier et Gastaldy, malgré le zèle qui les animait, n'ont jamais pu parvenir à avoir des renseignements que pour quelques pensionnaires. Très peu de malades sont dans le cas de les donner. Vous reconnaîtrez par l'habitude de la maladie que l'on est obligé d'aller longtemps en tâtonnant $»^{51}$.

L'étude du corpus de sources conservé dans les Archives départementales du Val de Marne ${ }^{52}$ permet de revisiter en partie ce conflit et plus généralement de découvrir les activités du directeur de l'hôpital de Charenton $^{53}$. Le conflit entre Coulmier et Royer-Collard est loin d'être anecdotique car il touche aux principes et aux pratiques du « traitement moral » ainsi qu'aux rapports de pouvoir au sein d'un établissement de santé dans un contexte de transformation théorique et sociale de l'espace médical $^{54}$. La Légion d'Honneur, que Coulmier reçoit sous l'Empire semble témoigner de la satisfaction des autorités... et pourtant.

\section{La question des registres et du contrôle des noms}

Dès les premiers jours de 1806, les demandes réitérées de RoyerCollard d'accéder aux registres des malades deviennent un objet de conflit. Ce dernier ne cesse en effet de justifier ses demandes par la volonté de mieux connaître ses malades et de pouvoir ainsi mieux les soigner :

« Je sais parfaitement, Monsieur, que ce n'est point à force de médicaments qu'on guérit l'aliénation et en vous demandant quelques détails sur les méthodes de traitement employés jusqu'ici dans l'hospice, j'étais loin de supposer que ces méthodes eussent dû consister dans une suite de moyens actifs. Mais il importe à votre humeur et au mien [sic] que l'hospice de Charenton ne soit pas tenu, quant à la partie médicale, avec moins

(51) «Lettre de Deguise », février 1806 [AN, AJ2 100/ AD Val-de-Marne, 2 Mi 56].

(52) En 1989, l'ensemble du fonds de la maison nationale de Charenton, conservé aux Archives nationales sous la cote AJ2 1-103, a été transféré aux Archives départementales du Val-de-Marne.

(53) Thierry HAUSTGEN, «Les débuts difficiles du Dr Royer-Collard à Charenton. État sommaire de la maison de Charenton sous le rapport du service médical et aperçu des réformes qui y sont nécessaires (1811), Antoine-Athanase Royer-Collard », Synapse, n 58, novembre 1989, p. 57-66 ; Idem, «La Maison de Charenton vers 1810, lieu de conflits institutionnels. Une lettre et un rapport confidentiel au ministre de l'Intérieur », L'Évolution Psychiatrique, 50, 1, 1985, p. 217-223.

(54) Il conviendrait ainsi de souligner que le «traitement moral n'est plus du tout du côté de la médecine clinique qui se constitue par ailleurs, à la fin de ce XVIII ${ }^{\mathrm{e}}$ siècle mais du côté d'une pratique de "direction morale" dont le médecin va s'emparer, sans y avoir été pourtant spécialement préparé » : dans Jacques Postel et Claude QueTEL (dir.), Nouvelle histoire de la psychiatrie, Paris, Dunod, 1994, p. 160. 
d'exactitude et de régularité que celui de la Salpêtrière et de Bicêtre ; et je sais de manière la plus positive qu'à la Salpêtrière, en particulier, on tient un état fort détaillé de tous les aliénés qu'on y reçoit [...]. Voilà ce que je désire faire à Charenton $»^{55}$.

Face aux refus successifs de Coulmier, Royer-Collard décide de créer ses propres registres, une initiative qui lui vaut la plus grande hostilité du directeur. Il ne cesse de justifier la nécessité thérapeutique d'enquêter sur les malades :

«Lorsque j'entrai en fonction, mon premier soin fut de vous prier de me faire donner un état exact de tous les malades qui existaient alors dans la maison avec l'indication de leur nom, de leur âge, de leur profession, de l'espèce de maladie dont ils étaient atteints, des causes auxquelles on en attribuait le développement et du traitement qu'ils avaient subi. Cet état me paraissait nécessaire pour agir avec connaissance de cause et rien en effet ne pouvait le suppléer. Vous eûtes la bonté de me le promettre à diverses reprises, mais soit défaut de temps soit défaut de zèle de la part de ceux que vous en aviez chargés, l'année s'est passée sans qu'il n'ait été fourni au moins complètement [À défaut d'obtenir ce registre] toutes les fois qu'un nouveau malade a été admis dans la maison, je l'ai interrogé sur son âge, sur sa profession, sur les diverses circonstances qui avaient précédé, accompagné et suivi l'invasion de la maladie. [...] Je me suis servi de toutes ces notes réunies pour tracer une histoire abrégée de chaque malade ; j'y ai suivi les maladies dans toutes leurs phases et depuis leur commencement jusqu'à leur terminaison. [Selon un membre de la maison] vous désireriez qu'il n'y eut point d'autre registre des malades que celui que vous faites tenir par l'administration de la maison ; vous voudriez qu' on ne me remit aucun état indicatif de leur entrée, de leur sortie, non plus que de leurs noms ; vous irez même jusqu'à exiger que je ne prisse aucune note sur leur personne ni aucun renseignement sur leur position, c'est-à-dire que je serai obligé de traiter des malades sans les connaître [...] qu'il me serait défendu de suivre leur histoire et de la rédiger, que je ne pourrais dans aucun temps revenir sur le passé et me rendre compte à moi-même de mes opérations $»^{56}$. 
En janvier 1807, Coulmier semble rechercher encore le compromis mais reste inflexible concernant son refus de communiquer au médecin les noms des malades :

«C'est précisément ce travail abrégé de chaque malade qui m'a effrayé si les noms s'y trouvaient et m'a fait prendre la ferme résolution de ne pas vous les communiquer. Ce tableau serait sans contredit instructif pour le traitement de la maladie, mais pourquoi dans un travail de ce genre mettre les noms des malades intéressants par eux et par les familles auxquelles ils appartiennent. Ne sont-ils assez malheureux par leur situation sans les exposer à voir leurs noms consignés dans un registre in-folio qui serait destiné à tracer le tableau des misères humaines. Il a, vous le savez, un cruel préjugé contre la maladie que l'on traite à Charenton, tout injuste qu'il soit, il n'en existe pas moins $»^{57}$. On peut interpréter les refus de Coulmier comme la marque d'un caractère «tyrannique » soucieux de conserver ses prérogatives administratives contre les empiètements du pouvoir médical. Derrière cette lutte autour des registres se dévoile une autre question, celle de l'identification des malades et de la préservation de leur vie privée. Le registre établi avant l'arrivée de Royer-Collard comprend 18 colonnes dont «1. La lettre initiale du nom du malade [en note : j'évite par ce moyen d'attacher publiquement le nom des familles à une maladie qui porte encore l'empreinte des faux préjugés] ; 2 . le sexe ; 3. l'âge ; 4. Le tempérament ; 5. la profession ; 6. L'invasion de la maladie ; 7. L'entrée dans la maison $[\ldots] \gg{ }^{58}$.

Selon Coulmier, il s'agit en effet de protéger le « secret » des familles et des malades, non pas aux yeux d'un médecin, mais plus généralement aux yeux d'un public contre lequel le malade doit être protégé.

Si les registres existent, ils doivent ainsi être gardés «en sûreté dans un greffe » et n'être consultés que « quand la nécessité l'exige ». Le risque, en autorisant Royer-Collard à enquêter et à tenir les registres, est de voir circuler le nom des malades et de mettre en cause le secret qui doit être garanti. S'il refuse de communiquer le nom des malades, Coulmier s'engage, pour aider le médecin, à communiquer le prénom, l'âge, la profession et le département d'origine du malade car « c'est les malades que vous devez traiter, non les noms. Ils vous sont inutiles et ce serait préjudiciable aux intérêts de la maison qui nécessairement perdrait

(57) Ibid., « Réponse de Coulmier à Royer-Collard » (31 janvier 1807)

(58) Ibid. 
la confiance de l'opinion publique ${ }^{59}$. Selon Jan Goldstein, l'attitude de Coulmier serait à replacer dans la continuité du respect ecclésiastique pour le secret de la confession ${ }^{60}$. On peut également rappeler que sous l'Empire, le refus des pratiques susceptibles de devenir les matériaux administratifs d'un renforcement d'un contrôle policier ne doit pas être négligé. En 1813, Coulmier justifie encore son refus d'imposer de nouvelles normes d'identification des malades :

« Je n'ai refusé à M. Royer-Collard qu'un nouveau registre qu'il demandait et désirait avoir chez lui en dépôt qui contiendrait les noms des malades, les prénoms, l'âge, le lieu de naissance, l'état de sa fortune, l'état de ses parents, le nom de sa femme, le nombre de ses enfants, questions dont la plupart ne pouvaient intéresser l'art de la médecine. Ce registre est un dépôt sacré $[\ldots]$ et ne doit pas sortir des archives de l'administration. Ce refus est fondé sur l'intérêt de la société et sur la demande de la plupart des parents qui redoutent le préjugé fâcheux que l'on a contre la folie que l'on pense héréditaire ce qui est un obstacle à beaucoup d'alliance dans un degré même éloigné ».

S'il est traditionnel de considérer les hospices et autres asiles comme des institutions d'État susceptibles de participer à l'enfermement des ennemis politiques, il convient aussi de constater que, sous la Révolution, certains de ces établissements ont servi de refuge à ceux qui, dans différents contextes, ont pu être inquiétés et poursuivis. Dirigé par l'abbé Sicard, l'Institut national des sourds et muets a ainsi accueilli des prêtres ou religieux réfractaires qui ont rejoint le personnel d'encadrement. Sous l'Empire, on peut penser que Coulmier cherche à éviter que les registres lui échappent afin de protéger certains malades, en particulier ceux qu'il désigne dans une des ses lettres comme les «prisonniers d'État», susceptibles d'être déplacés par les autorités dans des prisons sans doute beaucoup plus contraignantes. Il convient sans doute de considérer que la Maison de Charenton a pu être, sous l'administration de Coulmier, un refuge pour certains prisonniers, enfermés pour des raisons administratives et politiques, qui ont pu bénéficier de certaines libertés dont ils auraient été privés ailleurs. Il semble que c'est justement ce régime de liberté et la présence de ces prisonniers dans l'établissement que dénonce, au nom de la

(59) Ibid.

(60) Cf. Jan GoldSTEIN, «Foucault among the Sociologists: The "Disciplines" and the History of the Professions », History and Theory, Vol. 23, no. 2 (May, 1984), p. 170-192. Selon l'auteur, la médecine moderne serait, à l'inverse, à la recherche d'une totale transparence de l'individu. 
science médicale, Royer-Collard ${ }^{61}$. C'est d'ailleurs dans cette perspective que ce dernier déplace ses attaques contre Coulmier pour s'en prendre violemment à la présence du marquis de Sade au sein de l'hospice. À partir de 1808, les attaques du médecin se cristallisent en effet contre Sade. Royer-Collard instrumentalise les fantasmes dont ce dernier est déjà l'objet pour stigmatiser l'administration de Coulmier et dénoncer les aberrations thérapeutiques dont l'hôpital serait le théâtre.

\section{Sade à Charenton : un mythe?}

En 1808, la présence de Sade devient un moyen de fragiliser la position de Coulmier. Dans une lettre envoyée au ministère, Royer-Collard affirme qu' " il existe à Charenton un homme que son audacieuse immoralité a malheureusement rendu trop célèbre, et dont la présence dans cet hospice entraîne les inconvénients les plus graves. [...]. Cet homme n'est pas un aliéné. Son délire est celui du vice, et ce n'est point dans une maison consacrée au traitement médical de l'aliénation que cette espèce de délire peut être réprimée. [...] On a eu l'impudence de former un théâtre dans cette maison, sous prétexte de faire jouer la comédie par les aliénés, et sans réfléchir aux funestes effets qu'un appareil aussi tumultueux devait nécessairement produire sur leur imagination ». Ainsi, « il regarde comme un scandale public que cet homme immoral [Sade] dirige le petit théâtre que le directeur de l'hospice, Mr. de Coulmier, a cru devoir autoriser dans l'intérieur pour l'amusement et la distraction des malades $»^{62}$. Un fonctionnaire du ministère de l'Intérieur reprend les arguments du médecinchef de Charenton, dénonçant l'entière liberté dont bénéficie Sade et les dangers qu'il communique avec des détenus des deux sexes. À la fin de son rapport, ce médecin demande à faire déplacer Sade pour que les autres malades ne soient pas corrompus ${ }^{63}$. En septembre 1808, le préfet Dubois présente un rapport au ministre dans lequel il note qu'il « est vrai aussi qu'il est le maitre de déclamation des acteurs et actrices qui jouent la Comédie sur le Théâtre que le Directeur de l'hospice de Charenton a fait

(61) L'arrivée de Royer-Collard correspond d'ailleurs à ce que Michel Vovelle appelle la « grande lessive des détenus politiques » qui sont, au printemps 1807, soit relâchés, soit transférés dans les forteresses d'Ham ou d'If. Cf. Michel Vovelle, Théodore Desorgues, op. cit., p. 201.

(62) Cf. Détention du marquis de Sade [Lettre de Donatien-Alphonse-François, Mis de Sade. Rapports du Cte Louis-Nicolas-Pierre-Joseph Dubois et du Dr Antoine-Athanase Royer-Collard.] (Slnd).

(63) Michel Gourevitch, «Qui soignera le divin marquis ? Documents inédits sur les conflits de pouvoirs entre directeur et médecin à Charenton en 1812 », Perspectives psychiatriques, $1984,22, n^{\circ} 96$, p. $85-91$. 
élever. Ce directeur que j'ai fait appeler à ma préfecture, convient du fait, et dit même à cet égard qu'il a beaucoup d'obligation à de Sade parce que regardant la comédie comme un moyen curatif de l'aliénation d'esprit, il se trouve heureux d'avoir dans son hospice un homme capable de former à la scène des aliénés qu'il veut guérir par ce genre de remède. Ce n'est point à moi, mais aux médecins à décider si ce moyen curatif produit les résultats avantageux dont se félicite le Directeur de l'Établissement ». Dubois estime que, si la présence de Sade à Charenton constitue un scandale, il faut le transférer au château de Ham où il serait entretenu aux frais de sa famille ${ }^{64}$.

En juin 1810, alors que sa situation s'est affaiblie du fait du décès de son protecteur Thouret, Coulmier doit solliciter une entrevue avec le ministre de l'Intérieur qui semble avoir témoigné de son mécontentement lors d'une visite ( «j'ai été atterré lorsque je n'ai trouvé dans votre abord que l'expression du mécontentement après l'examen le plus sévère, n'ayant pas eu l'honneur d'être connu de vous, je n'ai pu attribuer cette défaveur qu'à quelque ennemi secret qui aurait tenté par de noires calomnies de me desservir auprès de vous ${ }^{65}$ ). Coulmier rappelle l'attention portée à l'amélioration des conditions de vie des patients et insiste sur l'approche philanthropique dans laquelle devait s'inscrire la pratique thérapeutique. Il réitère ses reproches contre Royer-Collard qui a toujours refusé d'adapter ses heures de visite aux rythmes de vie des malades: «M. le docteur, trouvant qu'il était pénible pour lui de faire les visites pendant les mois de novembre, décembre, janvier et février à 6 heures du matin, conformément au règlement, a désiré ne les faire qu'à 9 heures, heure qui n'est pas avantageux [sic] aux malades parce qu'alors l'heure pour distribuer les médecines et autres médicaments étant expirée, il fallait absolument remettre la distribution des remèdes au lendemain $»^{66}$.

Nommé inspecteur général des écoles de Médecine en 1809, RoyerCollard renforce à l'inverse, sa position au sein de la communauté médicale. Le 14 août, la Société médicale qu'il préside rejoint, sous l'impulsion du ministre de l'Intérieur, la Société académique de médecine présidée par Antoine Portal (1842-1832). À cette occasion, Royer-Collard prononce un discours particulièrement offensif contre la situation d'anarchie dans laquelle se trouve, selon lui, la médecine en France ${ }^{67}$. En 1812, des

(64) AN, F/7/6294, « Dossier Sade ».

(65) Ibidem, AJ2 100, « Minutes de lettre au ministre de l'Intérieur le 20 juin 1810 relativement à la visite qu'il a faite à Charenton ».

(66) Ibid., AJ2 100 [61-75], CoulmiER, « Précis sur la maison de santé du gouvernement à Charenton », 1 $^{\text {er }}$ septembre 1812.

(67) Bibliothèque médicale, Paris, 1810, t. XXX, p. 124-129. 
dénonciations de plus en plus nombreuses contre la gestion de Coulmier justifient l'intervention du Conseil d'État. Dès le mois de janvier, le ministre de l'Intérieur demande à un auditeur du Conseil d'État de se rendre à Charenton. Il faut attendre une année pour que Pepin de Belle-Isle, auditeur au Service extraordinaire du Conseil, commence son inspection. Coulmier intervient directement auprès des autorités pour tenter de répondre aux attaques de Royer-Collard. En mai 1812, il écrit à Barbier Neuville, chef de la $3^{\text {e }}$ division du ministère de l'Intérieur, un des protecteurs de Royer-Collard :

« J'ai cru que le temps pouvait affaiblir ce vif intérêt que vous portiez à M. Royer-Collard qui ne pouvait être fondé que sur les liaisons ou l'amitié que l'on conserve naturellement pour des compatriotes et ses alliés sans analyser ni les talents ni le zèle avec lequel on doit s'occuper des infortunés qui doivent trouver des secours dans l'art de la médecine. [Coulmier rappelle ses préventions lors de la nomination de Royer-Collard et le système mis au point pour organiser les visites] M. le médecin a eu la dissimulation de concentrer son mécontentement qui s'est accru par mon opposition à beaucoup d'innovations qu'il voulait faire, par mes représentations sur la manière dont les visites se faisaient, sur le temps qu'il employait à consulter plutôt les pensionnaires sur leurs noms, sur leurs pays, l'état et la fortune de leurs parents, questions curieuses qui ne pouvaient pas intéressées l'art de la médecine, sur des réflexions qu'il se permettait, souvent injurieuses et toujours pénibles à être entendues par des malades qui n'étaient pas dans un état de demence absolue. [Coulmier critique l'attitude de Barbier Neuville qui ne l'a jamais informé des accusations de Royer-Collard] votre air glacial et vos promesses évasives m'affligeaient au point que j'ai été forcé de prendre le parti de ne plus vous voir ni vous consulter $»^{68}$.

C'est dans ce contexte qu'est publiée la Notice sur l'hôpital de Charenton, une véritable charge particulièrement scandaleuse contre Coulmier rédigée par Hipollyte de Collins ${ }^{69}$. Ancien officier de cavalerie, Collins insiste particulièrement sur le lien entre Coulmier et Sade (« La première chose qui s'offre à mes regards est sa liaison intime avec un

(68) AN, AJ2 100 : «Lettre de Coulmier à Barbier-Neuville, le 10 mai 1812 relative à Royer-Collard

(69) Cette source a servi de vivier aux nombreux récits concernant la place occupée par Sade à Charenton. Cette notice a en effet été publiée pour la première fois en appendice au Journal inédit de Sade (Paris, 1970) 
monstre voué à l'exécration publique $\left.»^{70}\right)$. Collins dénonce surtout les effets du théâtre (avec gradins, loges et fosse d'orchestre qui se situait sous la salle des femmes aliénées) où seraient jouées les pièces de Sade : "Quelle confiance peut inspirer un Directeur auprès duquel on a souvent accès et faveur par un être auquel je ne puis donner le nom d'homme ; un Directeur qui consent à prendre un tel panégyriste ; qui fait jouer publiquement sur son théâtre une pièce faite par lui et à sa louange, dans laquelle on trouve les flatteries les plus basses et où on le compare aux dieux mêmes [...] J'ai vu tout un public frémir d'horreur à ce spectacle, tandis que le Directeur général rougissait de colère de n'entendre aucun applaudissement dans la salle $\gg^{71}$. Ces accusations, sans doute à l'origine d'un véritable mythe construit autour du théâtre de Sade à Chrenton, fragilisent d'autant plus Coulmier que ce dernier semble résister à certaines sollicitations des autorités administratives qui cherchent à renforcer la surveillance sur les patients, signe évident du rôle d'espace-refuge que pouvait jouer sous l'Empire la Maison de Charenton. Coulmier semble en effet refuser de répondre favorablement à certaines demandes émanant du ministère comme celle de rédiger un rapport sur un individu rattaché à l'administration de l'hôpital ${ }^{72}$. Coulmier prétend ne pas connaître l'individu en question, mais profite de l'opportunité pour dénoncer Royer-Collard et rédiger une lettre de délation contre lui :

« la grande inquiétude que j'éprouve d'après votre lettre provient du silence que j'ai gardé par délicatesse sur une personne dont les principes sont absolument opposés aux vues du gouvernement et surtout par la crainte que j'avais que l'on impute ma déclaration à un projet de vengeance contre un ennemi qui, depuis quatre ans, ne cesse de me calomnier et de me causer les plus cruels chagrins. [..] Je parle de M. Royer-Collard, docteur en médecine, homme d'un esprit très délié, adroit et insinuant, que les rapports que j'ai eu par ma place avec lui m'ont mis dans le cas d'apprécier et de pouvoir juger de ses principes politiques, qui a des relations très

(70) Hippolyte de CoLLINS, « Notice sur l'établissement consacré au traitement de l'aliénation mentale, établie à Charenton, près Paris, 1812 », Journal inédit de Sade, Paris Gallimard, Folio, 1994, p. 116.

(71) Ibidem

(72) AN, AJ2 100 : «Lettre de Montalivet à Coulmier », Paris, le 18 novembre 1812 : « J'aurais besoin, monsieur, de quelques renseignements sur la personne de M. David Delille, receveur de la maison de Charenton ; je vous prie de me faire connaître les relations sociales, l'influence qu'il exerce dans la société, son caractère, surtout son opinion politique et le degré de confiance qu'il vous inspire. Veuillez me donner le plus promptement possible ces renseignements qui seront pour moi seul et qui ne sortiront jamais de mon cabinet ». 
intimes dans vos bureaux, qui est un des inspecteurs de l'Université, place qui lui donne de l'influence sur les maîtres et les élèves. [...] Le vendredi 30 [octobre], [...] , Monsieur Royer-Collard, en parlant de la conspiration [de Malet] et de ses auteurs s'est permis de dire que l'événement qui venait de se passer, était la preuve la plus complète qu'il n'y avait en France ni gouvernement ni dynastie et que le tout reposait sur la pointe d'une aiguille. Son opinion sur le gouvernement pourra être répétée et confirmée par des personnes dignes de foi qui en ont été aussi indignées que moi $»^{73}$.

Dans cette lutte, Royer-Collard va s'appuyer sur l'affirmation de son autorité médicale pour écarter Coulmier.

\section{L'affirmation du pouvoir médical : la révolution terminée?}

Dans une lettre du 9 juillet 1813 de Royer-Collard, ce dernier reproche à Coulmier d'empiéter sur ses fonctions thérapeutiques. Pour Royer-Collard, il doit y avoir un partage des tâches, revendication qui s'appuie sur une décision ministérielle: «vous n'ignorez pas que le dernier arrêté de son excellence, le ministre de l'Intérieur, relatif à la maison de Charenton, porte expressément que le médecin en chef est exclusivement chargé de prescrire le régime que doivent suivre les malades et le traitement qui doit leur être administré ». Le médecin cherche à intervenir sur le placement des malades dans les différents quartiers : « le régime ne comprend-t-il pas nécessairement, ainsi que j'ai eu l'honneur de vous le rappeler dans ma lettre du 12 mai dernier, la détermination des lieux où doivent être placés les malades, le choix des personnes et des objets avec lesquels on peut les mettre en communication, et la mesure de liberté qu'il convient de leur accorder ?». À ces conflits de compétence s'ajoutent des enjeux concernant l'administration même de l'hôpital et, plus précisément, les « libertés » concédées aux malades :

« Il est encore un abus que vous entretenez, et qui doit cesser. Plusieurs malades ont la liberté, non seulement d'errer à leur gré dans l'intérieur de la maison, mais même de se promener seuls au dehors, au risque de s'y livrer à des extravagances et des troubles d'ordre public par des scènes désagréables. Je vous répète que c'est à moi à déterminer la mesure de liberté dont chaque malade peut jouir, et que vous ne devez par conséquent laisser sortir de la maison que ceux que j'en aurai jugés capables ».

(73) Ibid., «Lettre de Coulmier à son excellence monseigneur, le comte de Montalivet, ministre de l'Intérieur » (1812). 
À l'idéal de régénération issu de l'entreprise révolutionnaire auquel le traitement moral de Pinel donne corps succède un nouveau régime de savoirs qui transforme radicalement le regard sur l'aliéné qu'il s'agit désormais autant de contrôler que de soigner. Même si Esquirol continue le traitement moral lors de sa direction, la rupture avec le projet de Coulmier est bien nette. De plus en plus rapportée à des causes physiques ou physiologiques, la maladie est désormais interprétée en termes de dysfonctionnements singuliers. S'il s'agit toujours de s'inscrire dans le paradigme du physique et du moral, l'approche thérapeutique consiste ainsi à rétablir l'équilibre dans l'individu, le rôle du milieu ou de l'environnement passant dès lors au second plan. La promotion du rôle de la sociabilité ou des distractions dans le «traitement moral », telle que pouvait la défendre Coulmier, est désormais mise en cause en faveur d'une prise en charge médicale beaucoup plus individualisée, au risque naturellement de responsabiliser le malade sur le succès ou l'échec de la thérapie. De manière plus générale, la pratique thérapeutique n'est plus considérée comme participant à une logique d'inclusion politique, les possibilités de soigner et de transformer le fou en citoyen étant désormais présentées comme plus réduites. Dès lors, on s'interroge plus sur les conditions physiologiques ou anatomiques de la folie et sur la responsabilité judiciaire de l'aliéné que sur la possibilité de le perfectionner. Face à cette offensive, Coulmier rédige, en septembre 1812, une longue justification de son administration, véritable plaidoyer en faveur du traitement moral incarné par le médecin Gastaldy. Il rappelle certaines des activités thérapeutiques qu'ils proposaient aux malades :

« Nous cherchions à les dissiper par des jeux innocents, les concerts, la danse, des comédies dont les rôles étaient remplis par des malades, ce qui excitait entre eux une véritable émulation [...] Ces occupations les tenaient en activité, éloignaient les idées mélancoliques, source trop commune du délire. [...] Ce traitement moral approuvé par les personnes les plus respectables, par des étrangers qui sollicitaient avec empressement des billets d'entrées pour être témoins de l'influence des arts sur le moral comme sur le physique, était parvenu à établir une réputation à la maison de Charenton $[\ldots]$ ».

Coulmier mentionne encore le fait qu'il a accepté d'augmenter le traitement de Royer-Collard (1 000 francs), mais qu'en échange, ce dernier n'a pas fait plus de visites. Derrière les arguments scientifiques, une campagne de délation est menée contre le directeur comme en 
témoignent différentes lettres réunies aux Archives nationales ${ }^{74}$. Alors que les descriptions physiques de Coulmier servent alors à le stigmatiser (effet pervers des usages des rapports du physique et du moral) ${ }^{75}$, il semble que Royer-Collard suscite de faux témoignages pour perdre Coulmier : accusation de pédophilie, de tentative de viol, d'alcoolisme. Ainsi, un militaire, accusé de voler les autres détenus, accuse ce dernier des pires maux et finit sa lettre d'aveux adressée au ministre de la Police par cet appel au secours : «La seule grâce que je vous demande, Monseigneur, est de me faire transférer à Bicêtre, à Brest, à Rochefort, aux Isles Marguerite, à Missicipi [sic], au bout du monde, chez les anthropophages même, voulant absolument sortir de ce gouffre où triomphe le vice $\gg^{76}$. Les accusations de pédophilie et de viol, toujours justifiées par sa proximité avec Sade, ne font que salir davantage sa réputation. Coulmier tente de faire appel au public qui assistait à ces traitements ( « J'invoque le témoignage éclairé de tous les amis de l'humanité qui ont été témoins de la décence, de l'ordre établi dans la réunion des infortunés dont les chagrins étaient suspendus [...] par ce traitement moral ; celui des respectables mères de famille qui assistaient aux plaisirs innocents des pensionnaires et qui y conduisaient leurs enfants »). Le 30 mai 1814, Coulmier est démis de ses fonctions et est remplacé par l'administrateur Roulhac Dumaupas qui rédige, avec Royer-Collard, un nouveau règlement intérieur promulgué par le ministre de l'Intérieur en octobre 1814, quelques semaines avant la mort de Sade. Coulmier semble retrouver sa fonction lors des Cent Jours mais est définitivement renvoyé en 1815 .

\section{Pour une histoire politique de la folie à Charenton}

$\mathrm{Si}$ « l'enfer de Théodore Désorgues s'appelle l'oubli » ${ }^{77}$, celui de Coulmier s'appelle au contraire la mémoire construite au lendemain de sa défaite qui fixe pour longtemps sa mauvaise réputation. Les différentes notices biographiques, récits ou histoires rédigées dans les années 18201830 « par les vainqueurs » vont en effet canoniser de manière négative la figure de Coulmier. Entré en fonction comme directeur en 1826, Esquirol va fixer la légende noire de ce dernier en construisant une « image d'Épinal de

(74) Ibid., F15/2608 et 2609 (b).

(75) Le fait que Coulmier soit bossu et de petite taille suffit pour ses adversaires à assimiler ces « difformités » physiques aux «perversités » morales.

(76) Cité par Laure MURAT, L’homme qui se prenait pour Napoléon. op. cit., p. 156.

(77) Michel VovelLE, Théodore Désorgues, op. cit., p. 206. 
son asile ${ }^{78}$ dans son Mémoire historique et statistique sur la maison royale de Charenton ${ }^{79}$. Ces critiques se diffusent largement comme peut l'illustrer la notice publiée en 1836 dans la Biographie universelle ancienne et moderne des frères Michaud :

«De nombreuses plaintes s'élevèrent contre son administration, où il avait introduit un système très bizarre pour l'amusement, et même, disait-il, pour la guérison des aliénés. II leur procurait des spectacles de différents genres, réunissait souvent les deux sexes et laissait entrer dans la maison un grand nombre d'étrangers, ce qui avait donné lieu à d'incroyables abus. Coulmier lui-même, quoique d'un âge avancé, d'une constitution faible et d'une taille contrefaite, était fort relâché dans ses mœurs. Il s'était lié étroitement avec l'infâme de Sade, et cet homme odieux lui avait communiqué tous ses vices. Cet état de désordre dut cesser en 1814 à l'époque de la restauration et le directeur Coulmier perdit alors sa place. Se prétendant persécuté par les Bourbons, il y rentra en 1815, après leur départ ; mais il la perdit de nouveau après le second retour de Louis XVIII, et mourut dans l'obscurité le 4 juin $1818 »^{80}$.

Ces récits vont servir de matériaux aux historiens de la folie du $\mathrm{XX}^{\mathrm{e}}$ siècle. Les jugements négatifs portés sur Coulmier sont alors repris dans les travaux des étudiants de médecine ${ }^{81}$. Ils alimentent encore les imaginaires sur la folie dont la pièce de théâtre de Marat/Sade de Peter Weiss n'est qu' un exemple ${ }^{82}$. À leur tour, Gauchet et Swain érigent Coulmier

(78) Jeanne MESMIN D’EsTIENNE, «La folie selon Esquirol. Observations médicales et conceptions de l'aliénisme à Charenton entre 1825 et 1840 », Revue d'histoire du XIXe siècle, 2010/1, n40, p. 109

(79) Jean-Étienne ESQUIROL, Mémoire historique et statistique sur la maison royale de Charenton, Paris, Renouard, 1835. Au paragraphe «Autorité », Esquirol dénonce ainsi Coulmier : « Le ministre de l'intérieur, en rétablissant la maison de Charenton, fit une faute grave en se contentant de nommer les principaux chefs de l'établissement tels que le régisseur, le receveur, le médecin et le chirurgien, sans donner de règlement ni de mode de comptabilité, sans déterminer les attributions des divers fonctionnaires [...]. Il résulta de là que $\mathrm{m}$. de Coulmier fut administrateur absolu sous le nom, d'abord de régisseur, et puis sous celui de directeur. Le directeur percevait les revenus de la maison, sans rendre compte ; il administrait, disait-il, paternellement, nommant ou présentant à toutes les places, démolissant et bâtissant sans principes, ordonnant tout, se faisant obéir par tout le monde, depuis le dernier infirmier jusqu' au médecin en chef. [...] ». t. LXI, p. 463

80) « Coulmier », Biographie universelle ancienne et moderne, Paris, L.G. Michaud, 1836,

(81) Voir par exemple Adeline FRIDE, Charenton, ou la chronique de la vie d'un asile de la naissance de la psychiatrie à la sectorisation, Thèse pour le Doctorat de Troisième cycle en psychologie, G. LANTERI LAURA (dir.), 1983.

(82) La pièce de théâtre Marat/Sade : La Persécution et l'Assassinat de Jean-Paul Marat représentés par le groupe théâtral de l'hospice de Charenton sous la direction de Monsieur de Sade [ Die Verfolgung und Ermordung Jean Paul Marats dargestellt durch die Schauspielgruppe des Hospizes 
et son administration en symboles des archaïsmes médicaux et des erreurs politiques de la Révolution française. L'opposant systématiquement à Esquirol, incarnation à leurs yeux de la médecine « moderne » sur laquelle ils s'appuient ${ }^{83}$, ils ne cessent de critiquer l'expérience de Coulmier. Ils reprennent à leur compte, et sans aucune distance critique, les attaques les plus grossières adressées à ce dernier dans le contexte de luttes rappelées précédemment. Dès lors, Coulmier et son administration sont ravalés au rang d'un archaïsme teinté plus ou moins de charlatanisme ${ }^{84}$. Nos deux auteurs en viennent ainsi à réduire l'administration de Coulmier à une expérience extra-médicale. Ainsi, indifférents aux enjeux de la lutte entre l'administrateur et le médecin-chef, ils concluent qu'à « beaucoup d'égards, Coulmier semble avoir fondé le succès commercial de son entreprise sur l'exploitation des attitudes les plus traditionnelles en matière de folie (à commencer par l'intérêt pour le spectacle des fous) $\aleph^{85}$. Charenton et Coulmier vont encore occuper une place de choix dans l'ouvrage de Laure Murat qui consacre à son tour un long développement au séjour de Sade à Charenton. S'écartant de l'interprétation de Gauchet et de Swain, elle insiste particulièrement sur les relations privilégiées entre le "divin marquis » et l'ancien député à la Constituante en mettant en avant une proximité générationnelle, sociale et culturelle entre les deux acteurs, proximité qui devrait rendre compte de l'hostilité dont ils ont été tous les deux les cibles. L'appartenance à une certaine «culture » du XVIII siècle, largement idéalisée par l'auteur, devrait ainsi rendre compte du caractère subversif des deux hommes confrontés à la mise en ordre morale de l'Empire :

« La grande originalité qui singularise Charenton, c'est bien le théâtre, auquel le nom de Sade donne des proportions sataniques. Je serais tentée de croire que le véritable scandale du théâtre de Charenton ne touchait pas tant aux questions thérapeutiques [...] l'intolérable, pour les fonctionnaires de l'Empire et leurs successeurs, c'était l'ouverture, dans le monde forclos de l'asile, que représentait cette "autre scène", libre de tout contrôle

\footnotetext{
zu Charenton unter Anleitung des Herrn de Sade] retrace le passage à Charenton du marquis de Sade. Elle a été écrite entre 1961 et 1963 (représentée pour la première fois au Schillertheater de Berlin en 1964) et traduite en français par Jean Baudrillard aux éditions du Seuil en 1965 (théâtre Sarah Bernard en 1966). Coulmier y apparaît comme le représentant de l'autorité et de la raison (l'envers de Sade). Cette pièce a été portée à l'écran en 1966 par Peter Brook (neuf ans avant le Vol au-dessus d'un nid de coucou de Milos Forman).

(83) Les deux auteurs mettent particulièrement en valeur les pratiques thérapeutiques mises en œuvre par Esquirol dans la maison de santé pour les aliénés qu'il fonde en 1802 rue de Buffon. 63 et p.67 (84) Marcel GAUCHET et Gladys SWAIN, La pratique de l'esprit humain [2007], op. cit., p.

(85) Ibidem, p. 67, note 34
} 
idéologique, où Sade réhabilitait ce que la fête révolutionnaire et la propagande impériale, dans la raideur abstraite de leurs poses néoclassiques, avaient concouru à écraser : la matérialisation des corps et la circulation du désir $»^{86}$.

Cette approche permet à l'auteur de réhabiliter deux figures qui apparaissent ainsi plutôt sympathiques, mais éclaire-t-elle vraiment sur les raisons de l'éviction de Coulmier et sur les enjeux politiques qui entourent l'histoire de Charenton entre le Directoire et l'Empire ? Faute de sources, il est impossible de confirmer le rôle joué par Sade et le théâtre à Charenton... Faut-il voir dans le théâtre de Sade à Charenton un «mythe » construit pour salir la réputation de Coulmier ? Sous couvert d'écrire une histoire politique de la folie, Laure Murat, faute d'être réellement attentive au contexte et aux logiques politiques qui s'affrontent dans cette affaire, prêtant à l'inverse trop d'importance à des éléments qui participent plus de l'écriture romanesque (pensons à l'importance qu'elle accorde aux descriptions physiques des personnages !), en vient au contraire à proposer une lecture esthétisante et donc dépolitisée et désidéologisée de la folie. Alors que, derrière les condamnations lancées contre les pièces de Sade et la lutte qui oppose Coulmier au médecin Royer-Collard, il est possible de voir un épisode majeur de la mise en ordre impériale qui tourne le dos aux idéaux révolutionnaires et républicains, Murat invite le lecteur à lire cet épisode comme l'illustration de la transition entre un XVIII ${ }^{\mathrm{e}}$ siècle aristocratique et libertin à un $\mathrm{XIX}^{\mathrm{e}}$ bureaucratique et bourgeois :

« ces deux hommes du XVIII ${ }^{\mathrm{e}}$ siècle, marqués par les privilèges de la naissance, conservent de l'ancien monde une vision cosmique, interne, nourrie de fantaisie, dans le sens fort du mot, à l'intérieur de deux notions que la Révolution a congédiées : le plaisir et la volupté. Issus de l'âge du libertinage, ils partagent l'insoumission aux conventions, le goût, surtout, du théâtre ${ }^{87}$.

Si Coulmier a été finalement victime des attaques particulièrement violentes de Royer-Collard, ce n'est pas parce qu'il représentait la culture aristocratique et mondaine du XVIII ${ }^{\mathrm{e}}$ siècle mais parce qu'il incarnait des principes et des valeurs issus des transformations politiques de la Révolution française dont il conviendrait de mieux mesurer l'empreinte 
dans cette histoire politique de la folie. Michel Foucault, en dépit des critiques dont il peut encore être l'objet, avait bien mesuré le rôle joué par cet événement dans l'histoire de la folie. À trop vouloir l'occulter, le risque est grand de donner l'illusion d'écrire une histoire politique de la folie en proposant finalement un simple catalogue des différents imaginaires politiques construits sur la folie. Si cette approche doit avoir sa place, elle ne saurait prétendre au rang d'histoire politique de la folie pour la simple et seule raison qu'elle serait fondée sur un travail d'archives et s'achèverait par une prise de position politique de l'auteur face aux dysfonctionnements du traitement de l'aliénation aujourd'hui. Sur ce terrain comme sur d'autres, il convient de s'efforcer de mieux connaître l'histoire politique et sociale de la Révolution française qui peut ainsi offrir un vrai laboratoire de réflexion et de questionnement pour l'historien de la folie et, plus largement, pour tous ceux qui s'interrogent sur les modalités et les effets politiques des opérations de qualification et de disqualification du social.

Jean-Luc CHAPPEY

Université de Paris I Panthéon-Sorbonne Ea 127 Institut d'histoire de la Révolution française/Ums 622 jlchappey@gmail.com 